\title{
Parallel Implicit Solution of Full Navier-Stokes Equations
}

\author{
V.Ü. Ünal ${ }^{1}$ and $\ddot{U}$. Gülçat ${ }^{2}$ \\ ${ }^{1}$ Physics Department \\ Yeditepe University \\ 81120, Kayışdağı, İstanbul, Turkey \\ vunal@yeditepe.edu.tr \\ ${ }^{2}$ Faculty of Aeronautics and Astronautics \\ Istanbul Technical University \\ 80626, Maslak, İstanbul, Turkey \\ gulcataitu.edu.tr
}

\begin{abstract}
Parallel implicit solution of the incompressible Navier-Stokes equations based on two fractional steps in time and Finite Element discretization in space is presented. The accuracy of the scheme is second order in both time and space domains. Large time step sizes, with CFL numbers much larger than unity, are taken. The Domain Decomposition Technique is implemented for parallel solution of the problem with matching and non-overlapping sub domains. The segragate solution to tempereature field is obtained for the flow case where the forced convection is one order of magnitude higher than the free convection.
\end{abstract}

\section{Introduction}

As is well known, explicit schemes impose severe restrictions on the time step size for analyzing complex viscous flow fields, which are resolved with sufficiently fine grids. To remedy this, implicit flow solvers are used in analyzing such flows. Accuracy of the scheme is also a major issue in the numerical study of complex flows. Naturally, the higher order accurate schemes allow one to resolve the flow field with less number of grid points. Resolving the flow field with less number of points gives a great advantage to implicit schemes since the size of the matrix to be inverted becomes small.

In this study a second order accurate implicit scheme for solution of full NavierStokes equations is developed and implemented. The space is discretized with brick elements while modified version of the two-step fractional method is used in time discretization of the momentum equation. At each time step, the momentum equation is solved only once to get the half time step velocity field. The pressure, on the other hand, is obtained via an auxiliary scalar potential which satisfies the Poisson's equation. For the parallel implicit solution of the matrix equations, modified version of the Domain Decomposition Method, [1,2], is utilized, and direct inversion in each domain is performed. Super linear speed ups were achieved [3]. 
The implicit formulation for the momentum equation and the parallel implementation of the momentum, the pressure and the energy equation will be given in the following sections. The parallel solutions results obtained for the forced cooling of a room with chilled ceiling will be given at the end.

The scheme here is made to run on SGI Origin 3000 utilized with 8 processors running Unix operating system. Public version of the Parallel Virtual Machine, PVM 3.3, is used as the communication library.

\section{Formulation}

\subsection{Navier-Stokes Equations}

The flow of unsteady incompressible viscous fluid is governed with the continuity equation, [4],

$$
\nabla . \mathbf{u}=0
$$

the momentum (Navier-Stokes) equation

$$
\frac{\mathrm{D} \mathbf{u}}{\mathrm{Dt}}=-\nabla p+\frac{1}{\mathrm{Re}} \nabla^{2} \mathbf{u}+\frac{\mathrm{Gr}}{\operatorname{Re}^{2}} \mathrm{~T} \mathbf{k}
$$

and the energy equation

$$
\frac{\mathrm{DT}}{\mathrm{Dt}}=\frac{1}{\operatorname{RePr}} \nabla^{2} \mathrm{~T} .
$$

The equations are written in vector form (here on, boldface type symbols denote vector or matrix quantities). The velocity vector, pressure, temperature and time are denoted by $\mathbf{u}=\mathbf{u}(\mathrm{u}, \mathrm{v} . \mathrm{w}), p, \mathrm{~T}$ and $\mathrm{t}$, respectively. The variables are non-dimensionalized using a reference velocity and a characteristic length. Re is the Reynolds number, $\mathrm{Re}=\mathrm{U} H / \mathrm{v}$ where $\mathrm{U}$ is the reference velocity, $H$ is the characteristic length and $\mathrm{V}$ is the kinematic viscosity of the fluid. Gr represents the Grashof number, $\mathrm{Gr}=g \beta \Delta T H^{3} / v^{2}$, where $\beta$ is the coefficient of volumetric expansion. Pr denotes Prandtl number and $\mathbf{k}$ denotes the unit vector in $\mathrm{z}$ direction.

\subsection{FEM Formulation}

The integral form of Eq. (2) over the space-time domain reads as

$$
\iint_{\Omega \mathrm{t}} \frac{\partial \mathbf{u}}{\partial \mathrm{t}} \mathbf{N} \mathrm{d} \Omega \mathrm{dt}=\iint_{\Omega \mathrm{t}}\left(-\mathbf{u} \cdot \nabla \mathbf{u}-\nabla p+\frac{1}{\operatorname{Re}} \nabla^{2} \mathbf{u}+\frac{\mathrm{Gr}}{\operatorname{Re}^{2}} \mathrm{~T}^{\mathrm{n}} \mathbf{k}\right) \mathbf{N} \mathrm{d} \Omega \mathrm{dt}
$$


where $\mathbf{N}$ is an arbitrary weighting function. The time integration of both sides of Eq. (4) for half a time step, $\Delta \mathrm{t} / 2$, from time step $\mathrm{n}$ to $\mathrm{n}+1 / 2$ gives

$$
\begin{aligned}
& \int_{\Omega}\left(\mathbf{u}^{\mathrm{n}+1 / 2}-\mathbf{u}^{\mathrm{n}}\right) \mathbf{N} \mathrm{d} \Omega \\
& =\frac{\Delta \mathrm{t}}{2} \int_{\Omega}\left(-\mathbf{u} \cdot \nabla \mathbf{u}^{\mathrm{n}+1 / 2}-\nabla p^{n}+\frac{1}{\operatorname{Re}} \nabla^{2} \mathbf{u}^{\mathrm{n}+1 / 2}+\frac{\mathrm{Gr}}{\operatorname{Re}^{2}} \mathrm{~T}^{\mathrm{n}} \mathbf{k}\right) \mathbf{N} \mathrm{d} \Omega .
\end{aligned}
$$

At the intermediate time step the time integration of Eq. (4), where the convective and viscous terms are taken at $n+1 / 2$ and pressure term at time level $n$, yields

$$
\int_{\Omega}\left(\mathbf{u}^{*}-\mathbf{u}^{\mathrm{n}}\right) \mathbf{N} \mathrm{d} \Omega=\Delta \mathrm{t} \int_{\Omega}\left(-\mathbf{u} \cdot \nabla \mathbf{u}^{\mathrm{n}+1 / 2}-\nabla p^{\mathrm{n}}+\frac{1}{\operatorname{Re}} \nabla^{2} \mathbf{u}^{\mathrm{n}+1 / 2}+\frac{\mathrm{Gr}}{\operatorname{Re}^{2}} \mathrm{~T}^{\mathrm{n}} \mathbf{k}\right) \mathbf{N} \mathrm{d} \Omega .
$$

For the full time step, the averaged value of pressure at time levels $n$ and $n+1$ is used to give

$$
\begin{aligned}
\int_{\Omega}\left(\mathbf{u}^{\mathrm{n}+1}-\mathbf{u}^{\mathrm{n}}\right) \mathbf{N d} \Omega & =\Delta \mathrm{t} \int_{\Omega}\left(-\mathbf{u} \cdot \nabla \mathbf{u}^{\mathrm{n}+1 / 2}+\frac{1}{\operatorname{Re}} \nabla^{2} \mathbf{u}^{\mathrm{n}+1 / 2}\right. \\
& \left.-\nabla \frac{p^{\mathrm{n}}+p^{\mathrm{n}+1}}{2}+\frac{\mathrm{Gr}}{\operatorname{Re}^{2}} \frac{\mathrm{T}^{\mathrm{n}+1}+\mathrm{T}^{\mathrm{n}}}{2} \mathbf{k}\right) \mathbf{N} \mathrm{d} \Omega .
\end{aligned}
$$

Subtracting (6) from (7) results in

$$
\int_{\Omega}\left(\mathbf{u}^{\mathrm{n}+1}-\mathbf{u}^{*}\right) \mathbf{N} \mathrm{d} \Omega=-\frac{\Delta \mathrm{t}}{2} \int_{\Omega} \nabla\left(p^{\mathrm{n}+1}-p^{\mathrm{n}}\right) \mathbf{N} \mathrm{d} \Omega-\frac{\Delta \mathrm{t}}{2} \int_{\Omega}\left(\mathrm{T}^{\mathrm{n}+1}-\mathrm{T}^{\mathrm{n}}\right) \mathbf{k N} \mathrm{d} \Omega .
$$

If one takes the divergence of Eq. (8), the following is obtained;

$$
\int_{\Omega} \nabla \cdot \mathbf{u}^{*} \mathbf{N} \mathrm{d} \Omega=-\frac{\Delta \mathrm{t}}{2} \int_{\Omega} \nabla^{2}\left(p^{\mathrm{n}+1}-p^{\mathrm{n}}\right) \mathbf{N} \mathrm{d} \Omega-\frac{\Delta \mathrm{t}}{2} \int_{\Omega} \frac{\partial}{\partial \mathrm{z}}\left(\mathrm{T}^{\mathrm{n}+1}-\mathrm{T}^{\mathrm{n}}\right) \mathbf{N} \mathrm{d} \Omega .
$$

Subtracting (4) from (5) yields

$$
\mathbf{u}^{*}=2 \mathbf{u}^{\mathrm{n}+1 / 2}-\mathbf{u}^{\mathrm{n}} \text {. }
$$




\subsection{Numerical Formulation}

Defining the auxiliary potential function $\varphi=-\Delta \mathrm{t}\left(p^{\mathrm{n}+1}-p^{\mathrm{n}}\right)$ and choosing $\mathrm{N}$ as trilinear shape functions, discretization of Eq. (5) gives

$$
\left(\frac{2 \mathbf{M}}{\Delta \mathrm{t}}+\mathbf{D}+\frac{\mathbf{A}}{\operatorname{Re}}\right) \mathbf{u}_{\alpha}^{\mathrm{n}+1 / 2}=\mathbf{B}_{\alpha}+p_{e} \mathbf{C}_{\alpha}+\frac{2 \mathbf{M}}{\Delta \mathrm{t}} \mathbf{u}_{\alpha}^{\mathrm{n}}+\frac{\mathrm{Gr}}{\operatorname{Re}^{2}} \mathbf{M} \mathrm{T}^{\mathrm{n}} \mathbf{k}
$$

where $\alpha$ indicates the Cartesian coordinate components $\mathrm{x}, \mathrm{y}$ and $\mathrm{z}, \mathbf{M}$ is the lumped element mass matrix, $\mathbf{D}$ is the advection matrix, $\mathrm{A}$ is the stiffness matrix, $\mathbf{C}$ is the coefficient matrix for pressure, $\mathbf{B}$ is the vector due to boundary conditions and $\mathbf{E}$ is the matrix which arises due to incompressibility.

Equation for the temperature, Eq. (3) is solved explicitly using:

$$
\mathbf{M} \mathrm{T}^{\mathrm{n}+1}=\mathbf{M} \mathrm{T}^{\mathrm{n}}-\frac{\Delta \mathrm{t}}{2}\left(\frac{\mathbf{A}}{\operatorname{Pr} \cdot \operatorname{Re}}+\mathbf{D}\right) \mathrm{T}^{\mathrm{n}}
$$

The discretized form of Eq. (9) reads as

$$
\frac{1}{2} \mathbf{A} \varphi=-\frac{1}{2} \mathbf{A}\left(p^{\mathrm{n}+1}-p^{\mathrm{n}}\right) \Delta \mathrm{t}=2 \mathbf{E}_{\alpha} \mathbf{u}_{\alpha}^{\mathrm{n}+1 / 2}-\frac{\Delta \mathrm{t}}{2} \frac{\mathrm{Gr}}{\mathrm{Re}^{2}}\left(\mathrm{~T}^{\mathrm{n}+1}-\mathrm{T}^{\mathrm{n}}\right)_{e} C_{\mathrm{z}}
$$

Subtracting Eq. (6) from Eq. (7) and introducing the auxiliary potential function $\varphi$, one obtains the following;

$$
\begin{aligned}
\mathbf{M u}_{\alpha}^{\mathrm{n}+1} & =\mathbf{M u}_{\alpha}^{*}+\frac{1}{2} \mathbf{E}_{\alpha} \varphi+\frac{\Delta \mathrm{t}}{2} \frac{\mathrm{Gr}}{\mathrm{Re}^{2}}\left(\mathrm{~T}^{n+1}-\mathrm{T}^{n}\right) \\
& =2 \mathbf{M u}_{\alpha}^{\mathrm{n}+1 / 2}-\mathbf{M u}_{\alpha}^{\mathrm{n}}+\frac{1}{2} \mathbf{E}_{\alpha} \varphi+\frac{\Delta \mathrm{t}}{2} \frac{\mathrm{Gr}}{\mathrm{Re}^{2}}\left(\mathrm{~T}^{n+1}-\mathrm{T}^{n}\right)
\end{aligned}
$$

The element auxiliary potential $\varphi_{e}$ is defined as

$$
\varphi_{e}=\frac{1}{\operatorname{vol}\left(\Omega_{\mathrm{e}}\right)} \int_{\Omega_{e}} \mathbf{N}_{\mathrm{i}} \varphi_{\mathrm{i}} \mathrm{d} \Omega_{e}, \quad \mathrm{i}=1, \ldots \ldots \ldots, 8,
$$

where $\Omega$ is the flow domain and $\mathbf{N}_{\mathrm{i}}$ are the shape functions.

The following steps are performed to advance the solution one time-step.

i. Eq. (11) is solved to find the velocity field at time level $n+1 / 2$ with domain de composition,

ii. Eq. (12) is solved explicitly and temperature field $T^{\mathrm{n}+1}$ is obtained,

iii. Knowing the half step velocity field and the temperature, Eq. (13) is solved with domain decomposition to obtain the auxiliary potential $\varphi$.

iv. With this $\varphi$, the new time level velocity field $\mathbf{u}^{\mathrm{n}+1}$ is calculated via Eq.(14). 
$\mathrm{v}$. The associated pressure field $p^{\mathrm{n}+1}$ is determined from the old time level pressure field $p^{\mathrm{n}}$ and $\varphi$ obtained in the second step.

The above procedure is repeated until the desired time level. In all computations lumped form of the mass matrix is used.

\section{Domain Decomposition}

The domain decomposition technique, [3,5,6,7], is modified and applied for the efficient parallel solution of the momentum, Eq. (11) and the Poisson's Equation for the auxiliary potential function, Eq. (13).

\subsection{For the Momentum Equation}

Initialization: The momentum, Eq. (11) is solved with a direct solution method, separately in each domain $\Omega_{\mathrm{i}}$ with boundary of $\partial \Omega_{\mathrm{i}}$ and interface $S_{j}$, with vanishing Neumann boundary condition on the domain interfaces,

At the beginning of each time level: $\mu^{k}=0$ and $a w^{k}=0$,

$$
\begin{array}{cc}
\overline{\mathbf{A}}_{\mathrm{y}_{\mathrm{i}}}=\mathrm{f}_{\mathrm{i}} \quad \text { in } \Omega_{\mathrm{i}}, \\
\mathrm{y}_{\mathrm{i}}=\mathrm{g}_{\mathrm{i}} \quad \text { on } \partial \Omega_{\mathrm{i}}, \\
\frac{\partial \mathrm{y}_{\mathrm{i}}}{\partial \mathrm{n}_{\mathrm{i}}}=(-1)^{\mathrm{i}-1} \mu^{k} \quad \text { on } S_{j}
\end{array}
$$

$\mu^{o}=\mu^{k}, w^{o}=\mathrm{g}^{o}$ and $\mathrm{g}^{o}=a w^{k}-\left(\mathrm{y}_{2}^{o}-\mathrm{y}_{1}^{o}\right) S_{j}$.

where subscript $k$ denotes the interface iteration level at which convergence is obtained, subscript $o$ stands for the initial interface iteration level at the each time level and $\overline{\mathbf{A}}=\frac{2 \mathbf{M}}{\Delta \mathrm{t}}+\mathbf{D}+\frac{\mathbf{A}}{\operatorname{Re}}$ in Eq. (11) and $\mathrm{y}_{\mathrm{i}}=\mathbf{u}_{\alpha}^{\mathrm{n}+1 / 2}$.

Unit Problem: Inside the interface iteration cycle, each slave starts to solve the unit problem using the direct solution, together with the initialized Neumann condition received from the master at the end of the initialization step.

$$
\begin{aligned}
\overline{\mathbf{A}} \mathrm{x}_{\mathrm{i}}^{n}=0 & \text { in } \Omega_{\mathrm{i}}, \\
\mathrm{x}_{\mathrm{i}}^{n}=0 & \text { on } \partial \Omega_{\mathrm{i}},
\end{aligned}
$$




$$
\frac{\partial \mathrm{x}_{\mathrm{i}}^{n}}{\partial \mathrm{n}_{\mathrm{i}}}=(-1)^{\mathrm{i}-1} w^{n} \quad \text { on } S_{j}
$$

Each slave sends its new computed interface values to the master processor at the end of the unit problem and master starts post-processing of the received data from the slaves. The interface iteration cycle starts up at the master processor. Master optimizes the interface nodal values of the unknowns using the following 'steepest descent' procedure.

Steepest Descent: Interface iteration cycle goes on until the master processor finds correct Neumann conditions at the interfaces, $\mu^{k}$, at the end of its post processing of the interface data received from the slave processors,

$$
\begin{gathered}
a w^{n}=\left(\mathrm{x}_{1}^{n}-\mathrm{x}_{2}^{n}\right) S_{j} \\
\mathrm{~g}^{n+1}=\mathrm{g}^{n}-\beta^{n} a w^{n} \quad w^{n+1}=\mathrm{g}^{n+1}+s^{n} w^{n} \\
\beta^{n}=\frac{\sum_{j S_{j}}\left|\mathrm{~g}^{n}\right|^{2} d s \quad s^{n}=\frac{\sum_{j S_{j}}\left|\mathrm{~g}^{n+1}\right|^{2} d s}{\sum_{j S_{j} S_{j}\left(\mathrm{~g}^{n}\right)^{2} d s}\left(a w^{n}\right) w^{n} d s}}{\mu^{n+1}=\mu^{n}-\beta^{n} w^{n}}
\end{gathered}
$$

where $n$ denotes the interface iteration level.

Convergence check: If $\left|\mu^{n+1}-\mu^{n}\right|<\varepsilon_{\varphi}=10^{-4} \quad$ then $\mu^{n+1}=\mu^{k}$

Finalization: Having obtained the correct Neumann boundary condition for each interface, the momentum equation, Eq. (11), is solved via the direct solution method at each sub-domain, together with the correct interface conditions,

$$
\begin{array}{rlrl}
\overline{\mathbf{A}}_{\mathrm{y}_{\mathrm{i}}} & =\mathrm{f}_{\mathrm{i}} & \text { in } \Omega_{\mathrm{i}}, \\
\mathrm{y}_{\mathrm{i}} & =\mathrm{g}_{\mathrm{i}} & \text { on } \partial \Omega_{\mathrm{i}}, \\
\frac{\partial \mathrm{y}_{\mathrm{i}}}{\partial \mathrm{n}_{\mathrm{i}}}=(-1)^{\mathrm{i}-1} \mu^{k} & \text { on } S_{j}
\end{array}
$$

\subsection{Parallel Implementation}

During parallel implementation, in order to advance the solution single time step, the momentum equation is solved implicitly with domain decomposition. Solving Eq. (11) 
gives the velocity field at half time level, which is used at the right hand sides of Poisson's Eq. (13), in obtaining the auxiliary potential. The solution of the auxiliary potential is obtained with again domain decomposition where an iterative solution is also necessary at the interface. Therefore, the computations involving an inner iterative cycle and outer time step advancements have to be performed in a parallel manner on each processor communicating with the neighboring one.

\section{Results and Discussion}

The flow in a room, given in Fig. 1, with a Reynolds numbers of 1000, based on $1 \mathrm{~m}$. length, inflow speed and the kinematic viscosity, is studied. The computational domain has the following dimensions: sides $x=4 \mathrm{~m}, \mathrm{y}=2 \mathrm{~m}$ (symmetry plane) and maximum height $\mathrm{z}=2.6 \mathrm{~m}$. The solution is advanced up to the dimensionless time level of 75 , where the steady state is reached, with time step of 0.025 . Computations are carried with the 6-domain partitioning grids with total number of $61 \times 31 \times 35$ nodes. The following dimensionless numbers are employed. $\operatorname{Re}=1000, \operatorname{Pr}=0.7, \mathrm{Gr} / \mathrm{Re}^{2}=0.01$. The elapsed time per time step per grid point is 0.0002 seconds.

$$
\begin{aligned}
& 0<\mathrm{x}_{c}<0.2: y_{c}=\frac{m}{p^{2}}\left(2 p x_{c}-x_{c}^{2}\right) \\
& \left.0.2<\mathrm{x}_{c}<1: y_{c}=\frac{m}{(1-p)^{2}}\left((1-2 p)+2 p x_{c}-x_{c}^{2}\right)\right)
\end{aligned}
$$

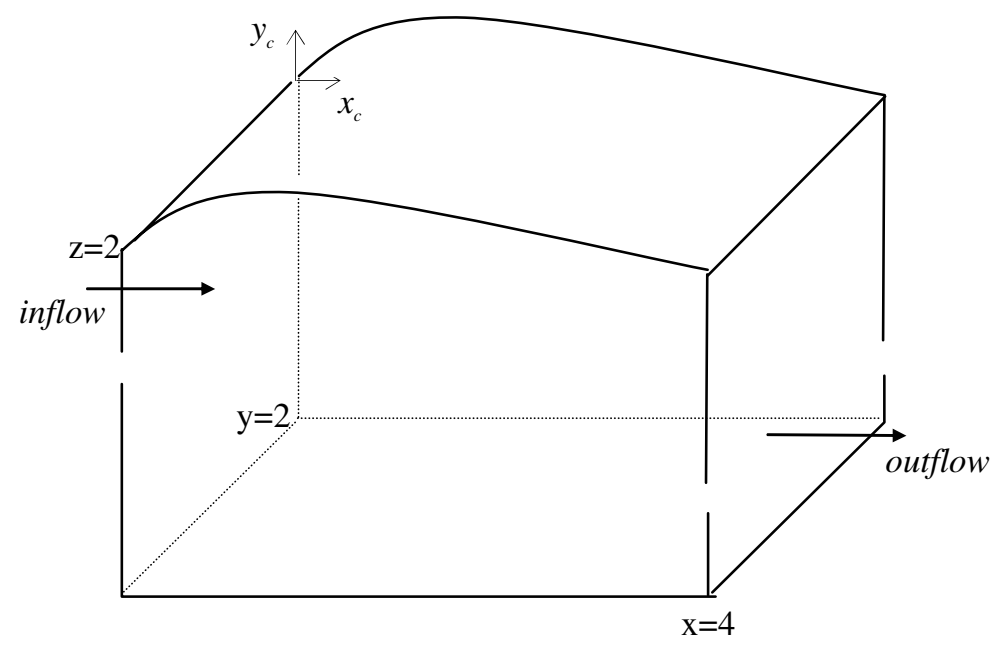

Fig. 1. Definition of the real problem 
Initial \& Boundary Conditions:

$\mathrm{u}=\sqrt{2} / 2, \mathrm{v}=0, \mathrm{w}=\sqrt{2} / 2$

$\mathrm{T}=-0.7 \quad$ at the top wall

$\mathrm{T}=\mathrm{T}(\mathrm{x})=(\mathrm{x}-2) / 4 \quad$ inside the cavity

$\mathrm{T}_{\mathrm{c}}=-0.5 \quad$ cold left wall

$\mathrm{T}_{\mathrm{h}}=0.5 \quad$ hot right wall

Computational grid with 6-domain partitioning (along the y-axis) is shown in Fig. 2. Velocity and temperature fields at the symmetry are presented in Fig. 3. The cooling effect of the chilled curved ceiling is obvious on the isotherm plots. The initially linear temperature field changes, because of the circulation in the room, so that the cool front moves towards the hot wall. Shown in Fig. 4 is the isotherms at $x=2$ plane where initially temperature is zero. Because of secondary currents in the room cool air packages are observed around the $\mathrm{x}=2$ plane.

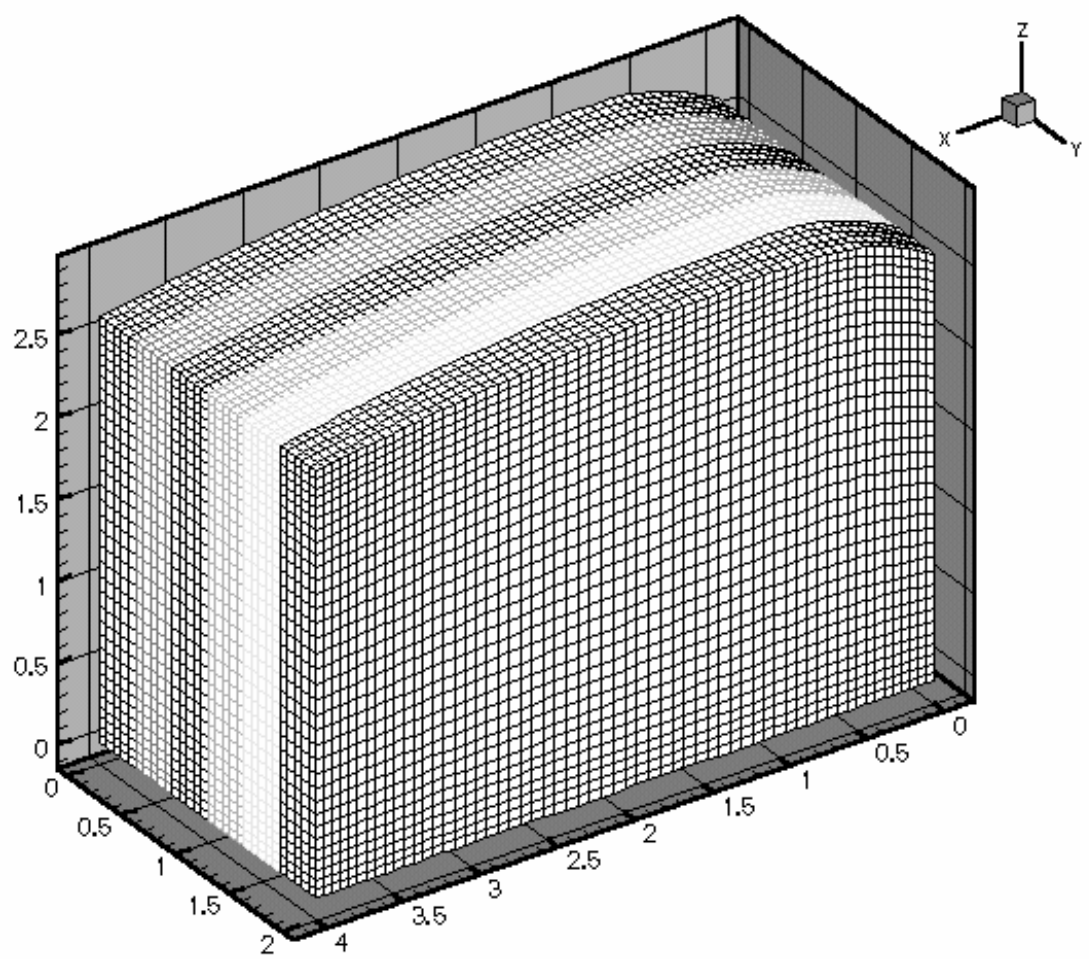

Fig. 2. 6-domain partitioning with total number of $61 \times 31 \times 35$ nodes 

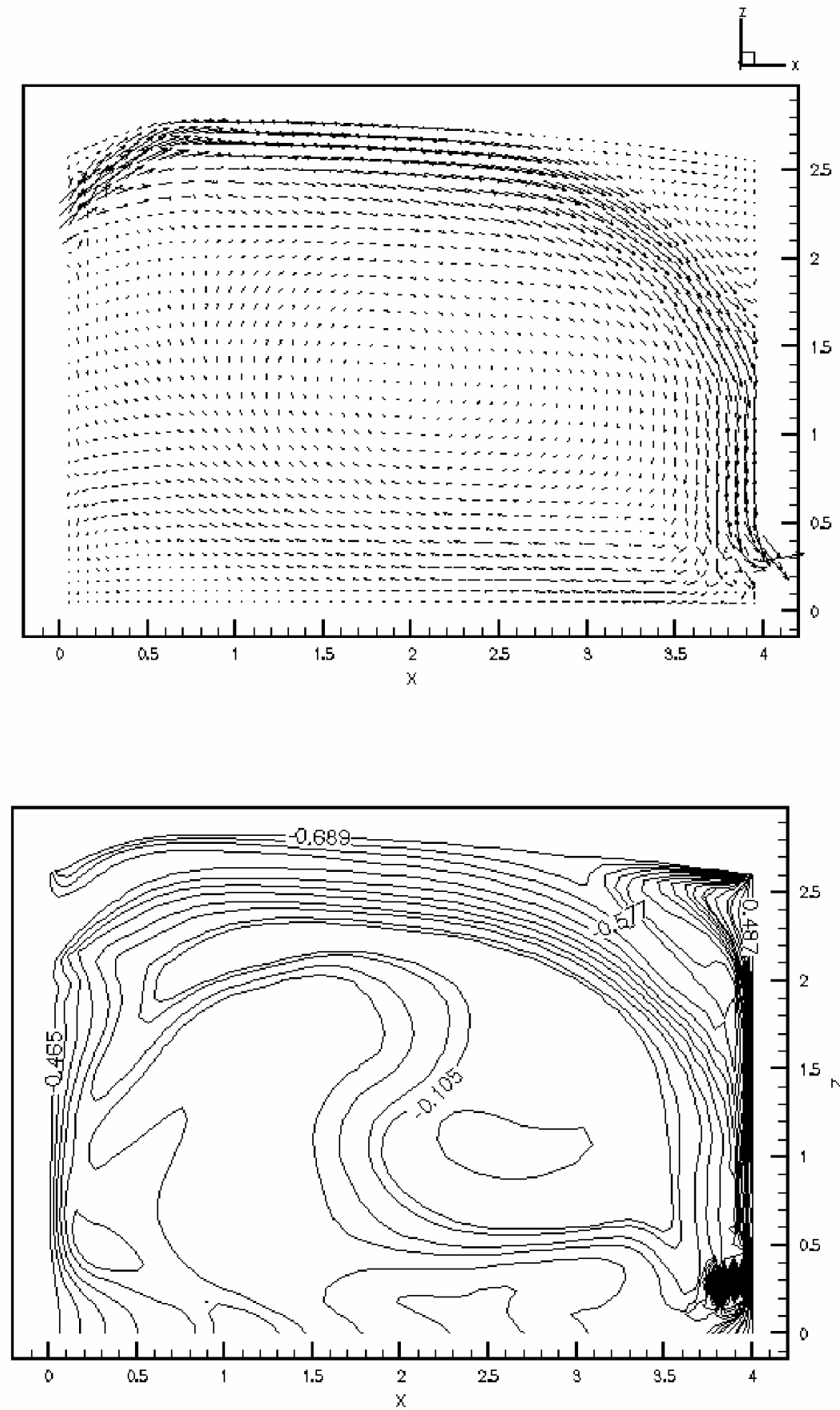

Fig. 3. Velocity \& temperature fields at $y=2$ symmetry plane, $\operatorname{Re}=1000, \operatorname{Pr}=0.7, \mathrm{Gr} / \operatorname{Re}^{2}=0.01$, $\Delta \mathrm{t}=0.025$ 


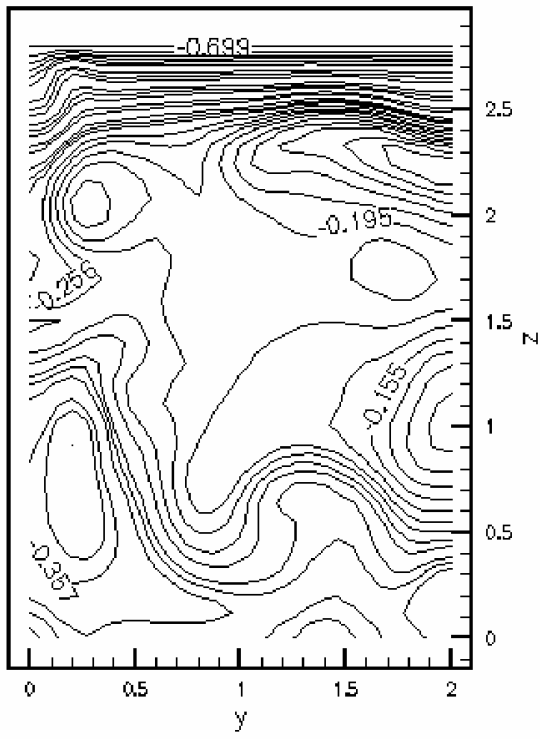

Fig. 4. Temperature field at $x=2$ plane, $\operatorname{Re}=1000, \operatorname{Pr}=0.7, \mathrm{Gr} / \mathrm{Re}^{2}=0.01, \Delta \mathrm{t}=0.025$

\section{References}

1. Gülçat, Ü. and Aslan, A.R., 1997. Accurate 3D Viscous Incompressible Flow Calculations with the FEM, International Journal for Numerical Methods in Fluids, 25, 985-1001.

2. Gülçat, Ü. and Üstoglu Ünal V., 2000. Accurate Implicit Solution of 3D Navier-Stokes Equations on Cluster of Workstations, Parallel CFD 2000 Conference, Trodheim, Norway, May 22-25.

3. Gülçat, Ü. and Üstoglu Ünal V., 2002. Accurate Implicit Parallel Solution of 3D NavierStokes Equations, Parallel CFD 2002 Conference, Japan, May 20-22.

4. Schlichting H., Boundary Layer Theory, McGraw-Hill, 1968.

5. Glowinski, R., and Periaux, J., 1982. Domain Decomposition Methods for Nonlinear Problems in Fluid Dynamics, Research Report 147, Inria, FRANCE.

6. Dinh, Q.V., Ecer, A., Gülçat, Ü., Glowinski, R., and Periaux, J., 1992. Applications to Fluid Dynamics, Parallel CFD 92, Rutgers University, May 18-20.

7. Glowinski, R., Pan, T.W., and Periaux, J., 1995. Parallel CFD 95. 